\title{
Anatomy of the lattice magnetic monopoles
}

\author{
V.G. Bornyakove, ${ }^{\mathrm{a}, \mathrm{b}}$, M.N. Chernodub ${ }^{\mathrm{c}}$, F.V. Gubarev ${ }^{\mathrm{c}, \mathrm{d}}$, \\ M.I. Polikarpov ${ }^{c}$, T. Suzuki ${ }^{\mathrm{e}}$, A.I. Veselov ${ }^{\mathrm{c}}$, V.I. Zakharov ${ }^{\mathrm{d}}$ \\ a Deutsches Elektronen-Synchrotron DESY 83 NIC, D-15735 Zeuthen, Germany \\ ${ }^{\mathrm{b}}$ Institute for High Energy Physics, Protvino 142284, Russia \\ c Institute of Theoretical and Experimental Physics, Moscow, 117259, Russia \\ d Max-Planck Institut für Physik, Föhringer Ring 6, 80805 München, Germany \\ e Institute for Theoretical Physics, Kanazawa University, Kanazawa 920-1192, Japan
}

\begin{abstract}
We study the Abelian and non-Abelian action density near the monopole in the maximal Abelian gauge of $S U(2)$ lattice gauge theory. We find that the non-Abelian action density near the monopoles belonging to the percolating cluster decreases when we approach the monopole center. Our estimate of the monopole radius is $R^{\text {mon }} \approx 0.04 \mathrm{fm}$.
\end{abstract}

\section{Introduction}

Confinement of color in QCD implies that the color field of external quarks is squeezed into a tube connecting the quarks (provided that the distance between the quarks is large enough). Similarly, the ordinary magnetic field cannot penetrate superconductors and the dual superconductor model of confinement [1] makes this analogy manifest. The model assumes condensation of magnetic monopoles in QCD, similar to the condensation of charged Cooper pairs in superconductor. The monopole confinement mechanism is confirmed in $S U(2)$ lattice gauge theory by many numerical calculations, for a recent review see, e.g., [2]. Microscopically, the condensation of the monopoles can be understood as percolation of a monopole cluster. And, indeed, it was observed that there exists always a big percolating cluster which is responsible for confinement [3]. Since the percolating cluster may seemingly have any size we will call it infrared (IR). On the other hand, there are also many small, or ultraviolet (UV) clusters which are usually viewed as lattice artifacts [3].

Although there is a lot of data on the lattice monopole the understanding of the monopole dynamics in terms of the continuum theory is far from being complete at the moment. Qualitatively, there are two ways of looking at the monopoles in non-Abelian theories. First, one can think in terms of an analogy with the 't Hooft-Polyakov monopoles [4] which are classical solutions to the Yang-Mills equations with a triplet of matter fields. However, there are no matter fields in QCD. As a result, one rather changes the strategy of defining the monopoles [5]. Namely, they can be defined as purely topological defects, with no direct relation to the density of the non-Abelian action. According to the original 
idea of Ref. [5] in case of $S U(2)$ gauge group one can choose any vector in color space and (partially) fix the gauge by rotating the vector to the third direction. Such gauge fixing fails when all the components of the vector vanish at some point. The crucial observation is that vanishing of a vector gives three conditions which in the $\mathrm{D}=4$ case define line-like defects, that is the monopole trajectories. The success of the monopole confinement model depends in fact on the particular choice of the gauge. The observation might imply that a purely topological definition, devoid of any dynamic content is not in fact adequate. The so called maximal Abelian gauge and the corresponding projection [2] turns out to be the most carefully studied and very successful. Since the Abelian projection emphasizes the role of the Abelian-like field configurations this might be an indication that at large distances the lattice monopoles are similar to the Abelian or Dirac monopoles.

To get insight into the dynamics of the lattice monopoles we will concentrate here on measuring the full non-Abelian and Abelian actions at the centers of the monopoles. Actually, this kind of measurements have been reported earlier [6]. Namely, it was shown that the non-Abelian action on the plaquettes close to the monopole trajectory is larger than the average plaquette action, $S$. It is easy to realize, however, that if this were true at arbitrary small distances the monopoles would be strongly suppressed by the action factor and could not condense, see, e.g., [7]. In this note we report on the measurements which demonstrate for the first time that the above mentioned excess of the action goes down for smaller lattice spacing, or larger $\beta$. A crucial novel point is that we distinguish between the monopoles belonging to the UV and IR clusters and the statement on the decreasing of the action refers to the IR monopoles only. In this sense, the structure of the IR and UV monopoles turns out to be different and one can say that the monopoles in the IR clusters are condensed due to their special anatomy.

The separation of the monopole ensemble on IR and UV clusters is unambiguous for large enough lattices. The distribution of the cluster lengths clearly shows [3] that each monopole configuration contains typically one large IR cluster and a lot of small UV clusters separated by clearly observed gap. Thus we do not need to introduce any artificial mass scale to distinguish between IR and UV clusters.

The most important question to be addressed here is the estimation of the size of the monopoles. Our definition of the monopole size will be described in Sect. 3 As we shall see, the size of the monopole turns out to be rather small numerically. This observation supports speculations on the existence of a numerically large mass scale in the non-perturbative physics, see, e.g., [8, 9] and references therein. On the other hand our results show that the size of the Abelian monopoles is much smaller than the distance between the monopoles. Thus the monopole cores are not overlapping and the system can be tractable as a dilute gas.

In the next section we will summarize the current views on the anatomy of the monopoles. In section 3 we present our data and discuss their implications. It occurs that due to the finite size the monopoles in gluodynamics are condensed at any value of the bare coupling. In compact QED, on the other hand, where monopoles are point-like, the critical coupling, separating confinement and deconfinement phases, exists. 


\section{Monopoles on the lattice and in the continuum}

Let us first remind the reader the backbone of the theory of the monopole condensation in the compact photodynamics [10]. In this case the monopoles are classical solutions, the same as the original monopoles of Dirac [11]. The radial magnetic field of the monopole is similar to the electric field of a point-like charge, $|\mathbf{H}| \sim 1 / e^{2} r^{2}$ where $e$ is the electric charge and the factor $1 / e^{2}$ appears because of the Dirac quantization condition. The corresponding energy is ultraviolet divergent:

$$
\epsilon_{m o n} \sim \int d^{3} x \mathbf{H}^{2} \sim \frac{1}{e^{2} a}
$$

where $\mathbf{H}$ is the magnetic field, $a$ is the lattice spacing which provides an ultraviolet cut

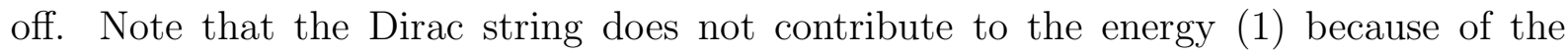
compactness of the $U(1)$. Otherwise it would result in a quadratically divergent term (for further details and references see [12]). Eq. (11) implies that the probability to find a monopole trajectory of length $L$ is suppressed by the action as $\exp \left\{-\right.$ const $\left.\cdot L /\left(e^{2} \cdot a\right)\right\}$. This suppression can be overcome, however, for $e^{2} \sim 1$ by the entropy factor. Indeed, on the hypercubic lattice the number $N$ of trajectories of the length $L$ grows exponentially, $N \sim \exp (\ln 7 \cdot L / a)$ where the constant $\ln 7$ is of pure geometrical origin. Since the self-energy (四) can be found with all the coefficients fixed the equating of the entropy and action factors provides a quantitative means to find the value of $e_{c r i t}^{2}$. A detailed quantitative analysis along these lines as well as further references can be found in [13].

In case of the gluodynamics, we choose the maximal Abelian gauge which is defined through maximization of the functional $R[U]=\sum_{l} \operatorname{Tr}\left[\sigma_{3} U_{l}^{+} \sigma_{3} U_{l}\right]$ over all gauge transformations $U_{l}^{\Omega}=\Omega^{+} U_{l} \Omega$. Moreover, in the standard parameterization of the link matrix

$$
U_{l}=\left(\begin{array}{cc}
\cos \varphi_{l} e^{i \theta_{l}} & \sin \varphi_{l} e^{i \chi_{l}} \\
-\sin \varphi_{l} e^{-i \chi_{l}} & \cos \varphi_{l} e^{-i \theta_{l}}
\end{array}\right)
$$

$\theta, \chi \in[-\pi,+\pi), \varphi \in[0, \pi)$, the functional $R$ can be rewritten as: $R[U]=\sum_{l} \cos 2 \varphi_{l}$.

Thus, the maximization of $R$ corresponds to the maximization of the absolute values of the diagonal elements of the link matrix (2). Since the $S U(2)$ plaquette action is $\beta \frac{1}{2} \operatorname{Tr} U_{P}$, at large values of $\beta$ the link matrices are close to unit matrix up to gauge transformations. Thus, at large values of $\beta$ in the maximal Abelian gauge $\cos \varphi_{l}$ are close to unity, the angles $\varphi_{l}$ are small and the $S U(2)$ plaquette action has the form:

$$
\beta S=\beta\left[\cos \theta_{P} \prod_{i=1}^{4} \cos \varphi_{i}+O\left(\sin \varphi_{l}\right)\right] .
$$

The projected action $S^{A b e l}$ is defined by putting $\varphi_{l}=0$. In the form (3) the projected action closely resembles the action of the compact electrodynamicsף:

$$
\beta_{U(1)} S_{c Q E D}=\beta_{U(1)} \cos \theta_{P} .
$$

\footnotetext{
${ }^{1}$ Here we neglect complications due to Faddeev-Popov determinant.
} 
However, if we would try to transfer the picture with Abelian monopoles directly onto the non-Abelian case, the conclusion would be that there is no monopole condensation in gluodynamics. Indeed, because of the asymptotic freedom $g^{2}(a) \rightarrow 0$ if $a \rightarrow 0$. Thus, one substantiates the dual superconductor model of the confinement with dynamical considerations like the following. Let us start increasing the lattice spacing a la Wilson. Then the corresponding effective coupling $g^{2}$ grows according to the renormgroup equations. The same coupling governs any of the $U(1)$ subgroups and once $g^{2}$ reaches the value where the $U(1)$ monopoles condense (see above) the condensation occurs in the non-Abelian theory as well. In this way one readily understands that there exists one monopole per volume of order $\left(\Lambda_{Q C D}\right)^{-3}$ so that the monopoles survive in the continuum limit. However, since the running of the coupling is a pure quantum effect there is no much hope to explicitly match a quasi-classical, Abelian-like field configuration at large distances with perturbative-vacuum fluctuations at short distances.

Instead, we can think in terms of a phenomenological expansion of the action density inside the monopoles. Since the action density is measured in lattice units it is convenient to consider an expansion of the form:

$$
\begin{aligned}
& \left(\left|F_{\mu \nu}^{a}\right|_{\text {monopole center }}^{2}-\left|F_{\mu \nu}^{a}\right|_{\text {average }}^{2}\right)= \\
& =\frac{1}{g^{2}(a) a^{4}}\left(\sum_{k=0}^{\infty} c_{k} g^{2 k}\left(a^{2}\right)+\sum_{n=1}^{\infty} b_{n} a^{n}\right)
\end{aligned}
$$

Then the theoretical expectation is that all the ultraviolet divergent pieces vanish, $c_{k}=0$. Indeed, otherwise we would have point-like objects beyond the ordinary gluons, in direct contradiction with the asymptotic freedom. Thus, only terms of order $\exp \left(-\right.$ const $\left./ g^{2}(a)\right)$ or powers of $a$ are allowed in the r.h.s. of Eq. (5). Moreover, we expect that the series actually starts with the $a^{4}$ term. Indeed, the monopole field is of order $\left(F_{\mu \nu}^{a}\right)_{m o n} \sim$ $\Lambda_{Q C D}^{2}$ as discussed above. The perturbative fields, on the other hand, are of order $a^{-2}$. However, there is no reason to expect any interference between the perturbative and monopole contributions, at least upon the averaging. Thus the excess of the action near the monopoles is to vanish proportional to $a^{4}$ if measured in the lattice units.

The prediction of the $a^{4}$ behavior holds in the academic limit $a \rightarrow 0$. It is a different matter of course how close to this limit the existing lattices are. In the next section we will present first indications that the excess, as measured in the lattice units, decreases with the decreasing lattice size. However, it is too early to claim that the excess is vanishing fast at $a \rightarrow 0$. In this sense the measurements presented in this paper can be considered as a first step in studying the monopole anatomy.

\section{Numerical results}

We have performed measurements of the full non-Abelian action, $S_{m o n}^{S U(2)}$, on the plaquettes closest to the monopole trajectory. The simulations have been done using Wilson action on lattices $12^{4}$ for $\beta=2.27,2.3,2.33,2.35,2.38,2.4,16^{4}$ for $\beta=2.45,20^{4}$ for $\beta=2.5,2.55$ and $28^{4}$ for $\beta=2.6$. We thus kept our physical volume $\gtrsim 1.5 \mathrm{fm}$. We made 


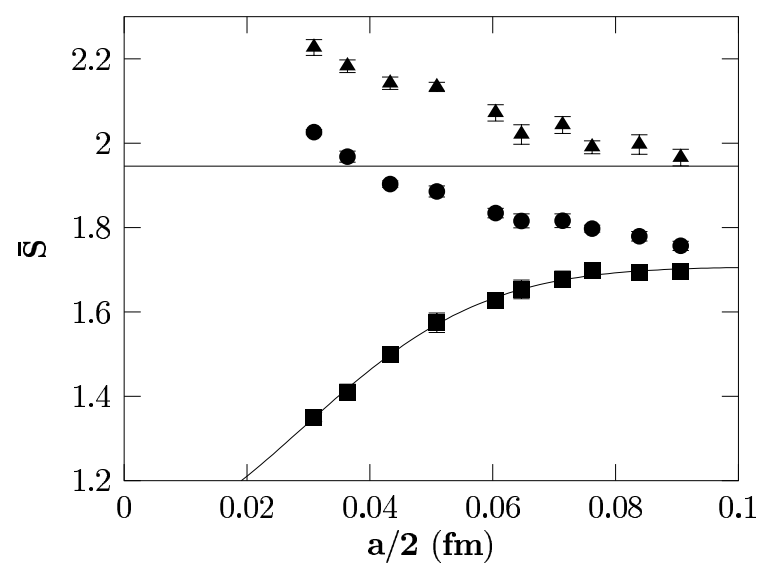

Figure 1: The dependence of excess of the non-Abelian action, $\bar{S}$, on the distance to the monopole, $a / 2$, for all monopoles (circles), monopoles from IR clusters (boxes) and monopoles from UV clusters (triangles). The dashed line is $\ln 7$. The error bars are within the symbols for most of points.

20 measurements on $12^{4}$ and $20^{4}$ lattices, 15 measurements on $16^{4}$ lattice and 20 measurements on $28^{4}$ lattice. The full non-Abelian action, $S_{m o n}^{S U(2)}$, on the plaquettes closest to the monopole trajectory have been measured. The error analysis has been carried out with bootstrap and jackknife methods. Both methods gave consistent estimates for statistical errors.

To fix MA gauge the simulated annealing (SA) algorithm was employed. It is known that this algorithm is vital for reducing the uncertainty due to Gribov copy effects in the gauge non-invariant observables computed in MA gauge [14]. Our SA algorithm implementation is essentially the same as described in [14 with the exception that we increased the total number of SA sweeps up to 2000. To further reduce bias due to Gribov copies we made gauge fixing for 5 randomly generated gauge copies for every Monte Carlo configuration. Only the copy with the maximal value of the gauge fixing functional $R[U]$ has been used to compute our observables. To estimate the residual effect of the Gribov copies we compared results obtained with different numbers of gauge copies $N_{\text {cop }}$ in the range from 1 to 5 . We have found systematic albeit weak dependence of our observables on the number of gauge copies. The difference between results obtained with $N_{\text {cop }}=1$ and $N_{\text {cop }}=5$ was less than our statistical errors. At the same time the difference from results obtained with the iterative gauge fixing algorithm was an order of magnitude larger than statistical errors.

While measuring $S_{\text {mon }}$ on the plaquettes closest to the monopole trajectory, we discriminate between the monopoles belonging to the IR and UV clusters. Our lattices are of the physical size $\gtrsim 1.5 \mathrm{fm}$, i.e. large enough for most of observables. On the other hand it has been found out in [3] that essentially larger volume is necessary to have only one large IR cluster. Some of our lattices are not large enough and we find on them not one but a few large clusters. We believe that combining all these clusters one gets the set of infrared monopoles. This conjecture has been confirmed recently [15]. In the present work 


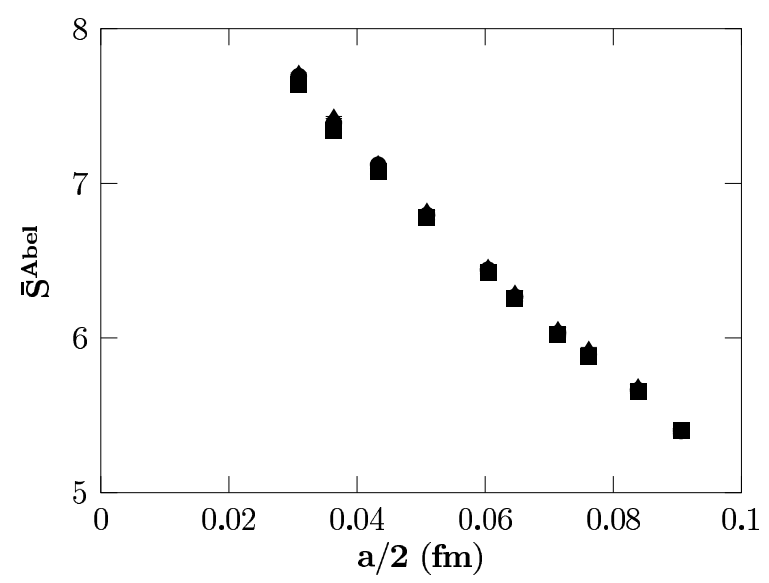

Figure 2: The same as in Fig. 11 for $\bar{S}^{\text {Abel }}$.

we considered only monopoles from the largest cluster as IR monopoles. This introduces some systematic uncertainty into results for UV clusters, namely the corresponding nonAbelian action is underestimated. At the same time this uncertainty does not influence our main conclusions.

In Fig. 11 we show the dependence of $\bar{S}=6 \beta\left\langle S_{\text {mon }}-S\right\rangle$ on the half of the lattice spacingf, $a / 2$. The factor $6 \beta$ is introduced here to make convenient the comparison of the action and the entropy factors. The explanation of the scale of the horizontal axis, i.e. $a / 2$, is the following. Since $\left\langle S_{m o n}\right\rangle$ is measured on the plaquettes which are faces of the cube dual to the monopole current, this corresponds to measuring the average field strength at the distance $a / 2$ from the monopole center. Note that the excess of the action is dominated by the closest plaquettes. In this figure we compare the action on the plaquettes nearest to the monopole center with the $\ln 7$. As is mentioned in Sect. 2, the $\ln 7$ is a geometrical constant determining the monopole entropy. The action in the lattice units for the percolating monopoles should not exceed $\ln 7$, see, e.g., [7, 13] and references therein.

Our main observation is that $\bar{S}$ for the monopole belonging to the IR cluster decreases when we approach the monopole center. Moreover, it is below $\ln 7$ for all data in agreement with percolation condition discussed above. On the other hand, the action for the UV monopoles is increasing and exceeds $\ln 7$ in agreement with the fact that these clusters are not percolating. Thus for the first time we demonstrate by direct computation of the action density that the percolation condition works in $S U(2)$ gluodynamics: percolating monopoles carry action density (in lattice units) less than $\ln 7$, while for non-percolating monopoles the action density is above this value. Note, that the excess of the action near all monopoles behaves similarly to the leading term in the monopole action [17]. This term is proportional to the length of the monopole trajectory.

The action density distribution on Fig. 1 has the same physical meaning as the action

\footnotetext{
${ }^{2}$ To define the lattice distance in Fermi, we find the correspondence between the bare charge and lattice spacing by fixing the value of the string tension $\sigma=440 \mathrm{MeV}$ and using the numerical data for the string tension in lattice units, $\sigma \cdot a^{2}$, see [16].
} 
profile of the 't Hooft-Polyakov monopole classical solution [四]. However the monopoles studied in the present Letter are of a purely quantum origin.

The results of the calculation of the Abelian action near the monopole, $\bar{S}^{\text {Abel }}=$ $6 \beta\left\langle S_{\text {mon }}^{\text {Abel }}-S^{\text {Abel }}\right\rangle$, are presented in Fig. 2. Unlike the full non-Abelian action, the Abelian action associated with the monopoles, $\bar{S}^{\text {Abel }}$, for monopoles belonging to IR and UV clusters is approximately the same. Moreover, it increases when one approaches the center of monopole. There is no known explanation of this effect. A comparison of Figs. 1 and 2 shows that the role of the off-diagonal degrees of freedom seem to compensate the divergent contribution into the monopole energy from the Abelian part of the gluon fields. Thus the monopoles in the maximal Abelian projection look like 't Hooft - Polyakov monopoles which are not singular at the origin. Note that the monopoles in compact QED are singular Dirac monopoles.

Now we discuss the size of the IR monopole. It occurs that the fit of the data in Fig. 11 by the function $C_{0}+C_{1} \exp \left\{-R^{2} /\left(R^{m o n}\right)^{2}\right\}, R=a / 2$, can be performed with high quality, $\chi^{2} / N_{D O F}=0.26$. The values of fit parameters are: $C_{0}=1.706(5), C_{1}=-0.63(2)$, $R^{m o n}=0.041(1) \mathrm{fm}$. This fit is shown in Fig. 1 by solid line. Thus our estimation for the monopole radius is $R^{\text {mon }} \approx 0.04 \mathrm{fm}$. Of course the definition of the monopole radius is not unique, but we believe that all reasonable definitions give the monopole radius of the same order. Note that in Ref. [7] it was found that the monopole condensation starts for monopoles approximately of the same physical size as $R^{\text {mon }}$ determined in the present paper. Up to now we did not study the scaling behaviour of the monopole radius. Such calculations are possible if we surround monopoles by cubes of various size and we measure the action on the faces of these cubes. This study is now in progress.

Our data give support to the following picture. At the large enough distances from the monopoles gauge field is Abelian-like and approximation eq. (3) works well. At short distances the non-Abelian nature of the monopoles is manifest and while the Abelian part of the action grows up the total action decreases.

To summarize, we have shown that the phenomenon of the monopole condensation in the lattice gluodynamics is due to a special anatomy of the monopoles belonging to the IR cluster. On the other hand, in the limit $a \rightarrow 0$ one would expect much faster vanishing of the excess of the action than it was observed so far. Since the theoretical prediction on the vanishing of the excess of the action at small $a$ seems very reliable (see the preceding section) the results obtained are to be rather interpreted in terms of various scales of the non-perturbative physics. Indeed, the average distance between nearest monopoles in the IR cluster is about $0.5 \mathrm{fm}$, as can be extracted from the data in Refs. [3, 15]. Now we observe for the first time that the excess of the action goes down when we approach the monopole center. The corresponding radius turns out to be small numerically" $R^{\text {mon }} \approx 0.04 \mathrm{fm}$. Moreover, even a smaller scale might emerge in future. Indeed, in the limit $a \rightarrow 0$ we should have the $a^{4}$ behavior for the excess of the action which is not yet in sight at present. Thus, there appears a hierarchy of scales all of which are formally of the same order, $\sim \Lambda_{Q C D}$. Note that existence of such hierarchies has already been conjectured on various grounds. First, a great variety of scales is manifested

\footnotetext{
${ }^{3}$ Note that this implies that monopoles form a gas rather than a liquid since the monopole cores are most probably not interacting due to the separation of scales.
} 
through QCD sum rules [8]. Further evidence has been accumulated via various lattice measurements, see 99 and references therein. In particular, very recently the relevance of the scale of order $2 \mathrm{GeV}$ was revealed through the measurements of the $\left\langle A^{2}\right\rangle$ vacuum condensate. This scale would roughly correspond to the monopole radius $R^{\text {mon }} \approx 0.04 \mathrm{fm}$ which we are observing.

\section{Acknowledgments}

M.I.P. and A.I.V. acknowledge the kind hospitality of the staff of the Institute for Theoretical Physics of Kanazawa University, where the work was initiated. Work of V.G.B., M.N.C., F.V.G., M.I.P. and A.I.V. was partially supported by grants RFBR 02-02-17308, RFBR 01-02-17456, INTAS 00-00111, JSPS Grant in Aid for Scientific Research (B) (Grant No. 10440073 and No. 11695029) and CRDF award RP1-2364-MO-02.

\section{References}

[1] G. 't Hooft, in High Energy Physics, ed. A. Zichichi, EPS International Conference, Palermo, (1975); S. Mandelstam, Phys. Rep. 23C, 245 (1976).

[2] R.W. Haymaker, Phys. Rept. 315, 153 (1999);

M. N. Chernodub and M. I. Polikarpov, "Abelian projections and monopoles", in "Confinement, duality, and nonperturbative aspects of QCD", Ed. by P. van Baal, Plenum Press, p. 387, hep-th/9710205.

[3] T.L. Ivanenko, A.V. Pochinsky, M.I. Polikarpov, Phys. Lett. B252, 631 (1990), Phys. Lett. B302, 458 (1993); S. Kitahara, Y. Matsubara, T. Suzuki, Prog. Theor. Phys. 93 (1995) 1; A. Hart, M. Teper, Phys. Rev. D58, 014504 (1998), Phys. Rev. D60, 114506 (1999).

[4] G. 't Hooft, Nucl. Phys. B190, 276 (1974);

A.M. Polyakov, JETP Lett. 20 (1974) 894.

[5] G. 't Hooft, Nucl. Phys. B190, 455 (1981).

[6] B.L.G. Bakker, M.N. Chernodub, M.I. Polikarpov, Phys. Rev. Lett. 80, 30 (1998); B.L.G. Bakker et al., Phys. Lett. B449, 267 (1999); H. Suganuma et al., Prog. Theor. Phys. Suppl. 131 (1998) 559; H. Ichie, H. Suganuma, Nucl. Phys. B574, 70 (2000).

[7] H. Shiba, T. Suzuki, Phys. Lett. B351, 519 (1995).

[8] V.A. Novikov et al., Nucl. Phys. B191, 301 (1981); K.G. Chetyrkin, S. Narison, V.I. Zakharov, ibid. B550, 353 (1999). 
[9] F.V. Gubarev, M.I. Polikarpov, V.I. Zakharov, Mod. Phys. Lett. A14 (1999) 2039;

A. G. Williams, "Gluons, quarks, and the transition from nonperturbative to perturbative QCD", hep-ph/0107029;

E.V. Shuryak, Scales and phases of nonperturbative QCD, hep-ph/9911244.

[10] A.M. Polyakov, "Gauge Fields and Strings", Harwood, New York, 1987.

[11] P.A.M. Dirac, Proc. R. Soc. London A133 (1931) 60.

[12] M.N. Chernodub et al., Nucl. Phys. B592, 107 (2000); Nucl. Phys. B600, 163 (2001);

[13] H. Shiba, T. Suzuki, Phys. Lett. B343, 315 (1995).

[14] G.S. Bali et al., Phys. Rev. D54, 2863 (1996); G.S. Bali et al., Nucl. Phys. Proc. Suppl. 42, 852 (1995); V.G. Bornyakov, D.A. Komarov, M.I. Polikarpov, Phys. Lett. B497, 151 (2001).

[15] V. Bornyakov and M. Muller-Preussker, Nucl. Phys. Proc. Suppl. 106, 646 (2002).

[16] J. Fingberg, U. M. Heller, F. Karsch, Nucl. Phys. B392, 493 (1993).

[17] T. Suzuki, Prog. Theor. Phys. Suppl. 131 (1998) 633. 